\title{
Thermoluminescent Properties of Undoped and Ce-Doped Lutetium Orthosilicate and Yttrium Orthosilicate Single Crystals and Single Crystalline Films Scintillators
}

\author{
Anna Twardak, Paweł Bilski, Yuriy Zorenko, Tanya Zorenko, Vitaliy Gorbenko, Ewa Mandowska, \\ Arkadiusz Mandowski, Oleg Sidletskiy, and Jiri Mares
}

\begin{abstract}
In this work the comparative analysis of thermoluminescent properties of undoped and Ce-doped single crystals (SC) and single crystalline films (SCF) of $\mathrm{Lu}_{2} \mathrm{SiO}_{5}$ (LSO) and $\mathrm{Y}_{2} \mathrm{SiO}_{5}$ (YSO) orthosilicates were performed. The $\mathrm{SC}$ samples were prepared with the Czochralski method, and SCF were grown by the liquid phase epitaxy technique. We show that such different methods of material preparation resulted in different thermoluminescent properties of undoped and $\mathrm{Ce}^{3+}$ doped LSO and YSO SC and SCF caused by the presence host defects (first of all, oxygen vacancies) as trapping centers in $\mathrm{SC}$ and formation of $\mathbf{P b}^{2+}-\mathrm{Ce}^{4+}$ pair centers in SCF.
\end{abstract}

Index Terms - LSO, single crystalline films, single crystals, thermoluminescence, YSO.

\section{INTRODUCTION}

$\mathbf{T}$ HERMOLUMINESCENCE (TL) is a phenomenon of light emission by an insulator or semiconductor, which can be observed when previously irradiated material is thermally stimulated. Trapping of charge carriers by electron traps leading to TL phenomenon is competitive to their trapping by

Manuscript received May 19, 2013; revised July 19, 2013 and August 02, 2013; accepted October 03, 2013. Date of publication January 09, 2014; date of current version February 06, 2014. This work was supported in part within the NATO project CBP.NUKR.CLG984305, Polish National Science Centre (NCN) projects No 2012/05/N/ST8/03334, No. 2012/07/B/ST5/02376, and No 2013/05/N/ST5/204583 and Ministry Education and Science of Ukraine project SL-126F.

A. Twardak and P. Bilski are with the Institute of Nuclear Physics, Polish Academy of Sciences, Krakow, Poland (e-mail: anna.twardak@ifj.edu.pl; pawel.bilski@ifj.edu.pl).

Y. Zorenko is with the Institute of Physics, Kazimierz Wielki University in Bydgoszcz, Bydgoszcz, Poland on leave from the Electronics Department, Ivan Franko National University of Lviv, Lviv, Ukraine (e-mail: zorenko@ukw.edu. pl).

T. Zorenko and V. Gorbenko are with the Electronics Department Ivan Franko National University of Lviv, Lviv, Ukraine (e-mail: tanyazorenko@gmail.com; horbenko@inbox.ru).

E. Mandowska and A. Mandowski are with the Institute of Physics, Jan Dlugosz University, Czestochowa, Poland (e-mail: e.mandowska@ajd.czest.pl; a.mandowski@ajd.czest.pl).

O. Sidletskiy is with the Institute for Scintillation Materials National AS of Ukraine, Kharkiv, Ukraine (e-mail: sidletskiy@isma.kharkov.ua).

J. Mares is with the Institute of Physics Academy of Science of Czech Republic, 16253 Prague, Czech Republic (e-mail: amares@fzu.cz).

Color versions of one or more of the figures in this paper are available online at http://ieeexplore.ieee.org.

Digital Object Identifier 10.1109/TNS.2013.2285288 luminescence centers acting in scintillation process. For this reason, the study of TL properties of materials is therefore complementary for scintillation research. It can provide additional information on trapping centers and hence contribute to a better characterization of a material.

The lutetium orthosilicate $\mathrm{Lu}_{2} \mathrm{SiO}_{5}$ (LSO) and yttrium orthosilicate $\mathrm{Y}_{2} \mathrm{SiO}_{5}$ (YSO) are well-known luminescent materials [1]. They are commonly used as scintillators, i.e., in positron emission tomography (PET) [2]-[4]. Apart from that, the LSO-based scintillating screens are competitive to other scintillating materials such as garnets (LuAG and GGG) and perovskites (LuAP) for X-ray imaging due to their host parameters. LSO host density is high $\left(\rho=7.4 \mathrm{~g} / \mathrm{cm}^{2}\right)$, and the same relates to its effective atomic number $\left(\mathrm{Z}_{\mathrm{eff}}=66\right)$. It provides a good spatial resolution of X-ray images [5]-[7].

LSO and YSO scintillators can be grown in form of single crystals (SC) using Czochralski method [2]-[4]. However, interesting scintillation properties can be achieved by growing them as single crystalline films (SCF) using liquid phase epitaxy (LPE) method [5]-[10]. Differences in conditions of SC and SCF growth processes results in the different concentrations of main type defects in them, first of all, oxygen vacancies [9]. Moreover, the different distribution of $\mathrm{Ce}^{3+}$ ions over positions of LSO and YSO host and influence of $\mathrm{Pb}^{2+}$ contamination coming from the flux used for the film growth also caused differences in luminescent properties of SC and SCF [8]-[10].

Existence of differences in thermoluminescent properties of LSO SC and SCF counterparts was reported in our previous work [11]. We showed that thermoluminescence glow curves strongly depend on crystalline form of the SC and SCF samples. In this paper, we perform more detailed comparison of the TSL properties of undoped and $\mathrm{Ce}^{3+}$ doped YSO and LSO SC and SCF for the explanation of the mentioned differences in the nature of their trapping centers.

\section{Materials AND MethodS}

In this work, 25 different samples were investigated: 14 LSObased samples and 11 YSO-based samples (see Table I). In both cases, samples in form of SCs and SCFs were available. The vast majority of them were doped with cerium (21 samples).

SCs were grown from melt by the Czochralski method in Ar atmosphere in the Institute for Scintillation Materials (ISMA) in 
TABLE I

INVESTIGATED Single CRYSTAL SAMPLES

\begin{tabular}{ccc}
\hline \hline Sample number & Sample content & $\begin{array}{c}\mathrm{Ce}^{3+} \text { ions content } \\
\text { at. \%) }\end{array}$ \\
\hline 16 & LSO & - \\
17 & LSO:Ce & $>0.01$ \\
18 & LSO:Ce & $\sim 0.01$ \\
19 & LSO:Ce & 0.027 \\
25 & YSO & - \\
11 & YSO & - \\
12 & YSO & - \\
26 & YSO:Ce & $>0.1$ \\
27 & YSO:Ce & 0.07 \\
\hline \hline
\end{tabular}

TABLE II

Investigated Single CRystalline Film SAMPLES

\begin{tabular}{ccccc}
\hline \hline $\begin{array}{c}\text { Sample } \\
\text { number }\end{array}$ & $\begin{array}{c}\text { Sample } \\
\text { content }\end{array}$ & $\begin{array}{c}\mathrm{CeO}_{2} \text { content } \\
\text { in MSs } \\
(\mathrm{mol} \%)\end{array}$ & $\begin{array}{c}\text { Ce content } \\
\text { in SCF } \\
(\text { at. } \%)\end{array}$ & $\begin{array}{c}\text { SCF } \\
\text { thickness } \\
(\mu \mathrm{m})\end{array}$ \\
\hline 1 & LSO & - & - & 9 \\
2 & LSO:Ce & 5 & $\sim 0.01$ & 15 \\
3 & LSO:Ce & 5 & 0.025 & 21 \\
4 & LSO:Ce & 10 & & 22 \\
5 & LSO:Ce & 10 & 0.02 & 11 \\
6 & LSO:Ce & 10 & & 10 \\
7 & LSO:Ce & 10 & 0.055 & 37 \\
8 & LSO:Ce & 20 & 0.04 & 13 \\
9 & LSO:Ce & 20 & 0.04 & 10 \\
10 & LSO:Ce & 20 & - & 13 \\
21 & YSO & - & 0.01 & 17 \\
22 & YSO:Ce & 5 & 0.02 & 15 \\
23 & YSO:Ce & 10 & 0.06 & 6 \\
24 & YSO:Ce & 22 & & 10 \\
56 & YSO:Ce & 22 & 0.05 & 10 \\
57 & YSO:Ce & 22 & & \\
\hline \hline
\end{tabular}

Kharkiv (see table I). Two of YSO SC samples (samples 11 and 12) are identical to the substrates for growth of LSO:Ce SCF samples (samples 8 and 9 , respectively).

SCFs were crystallized using the liquid phase epitaxy (LPE) method at the Electronic Department of National University of Lviv from the super-cooled melt-solution (MS) based on the $\mathrm{PbO}-\mathrm{B}_{2} \mathrm{O}_{3}$ flux [5]-[7]. Both LSO and YSO-based SCFs were grown onto undoped YSO substrates. The thicknesses of SCF were from several up to several tens of micrometers (Table II). For Ce-doped SCFs, the concentration of $\mathrm{CeO}_{2}$ activator oxide ions in melt solution was 5,10 , or $20 \mathrm{~mole} \%$ for the set of SCFs based on LSO and 5, 10, and $22 \mathrm{~mole} \%$ for YSO-based series. From microanalyses of the content of several LSO:Ce and YSO:Ce SCF samples performed using a JEOL JXA-8612 MX electron microscope, we have found that the $\mathrm{Ce}^{3+}$ content, depending on the $\mathrm{CeO}_{2}$ concentration in MS and temperature of SCF growth, was varied in the 0.01-0.055 at.\% range for LSO:Ce SCFs and in the 0.01-0.06 at.\% range for YSO:Ce SCFs. Therefore, the segregation coefficient of $\mathrm{Ce}^{3+}$ in these SCFs was very low and equal to about $0.002-0.0027$ for YSO:Ce SCFs and 0.002-0.00275 for LSO:Ce SCFs.

For comparison, samples of lithium fluoride doped with magnesium, copper, and phosphorus $\mathrm{LiF}: \mathrm{Mg}, \mathrm{Cu}, \mathrm{P}(\mathrm{MCP})$ were used. MCP is commonly applied thermoluminescent material, which is highly sensitive to ionizing radiation [12], [13].

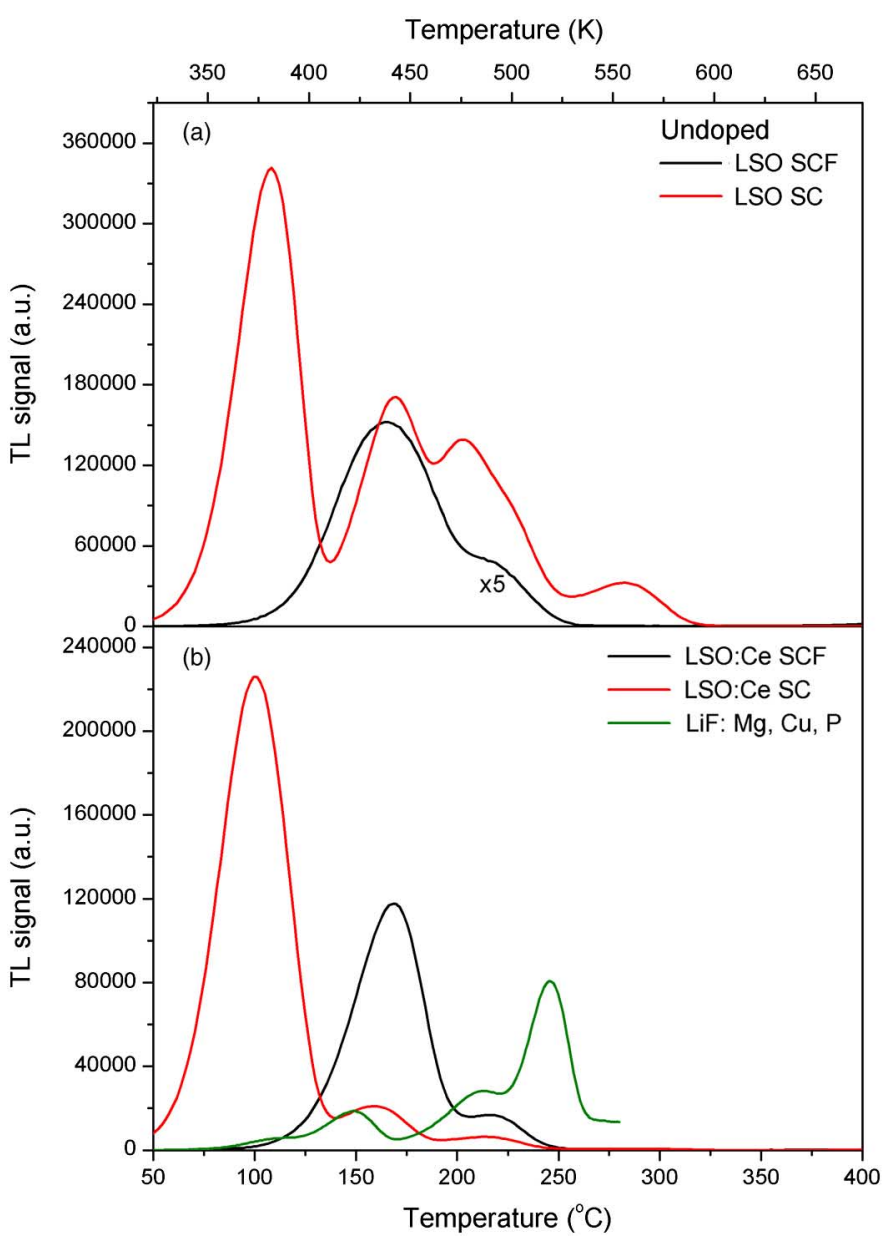

Fig. 1. Glow curves of undoped and Ce-doped LSO SC and SCF after irradiation with alpha particles. All glow curves were normalized to unit mass of alpha penetration layer $(1 \mathrm{mg}$ ) and unit mean dose (1Gy). TL signal of LSO SCF were multiplied by factor 5. (a) LSO SC (sample 16) and LSO SCF (sample 1). (b) LSO:Ce SC (sample 19) and LSO:Ce SCF (sample 6).

The thermally stimulated luminescence was measured using a Risoe DA-20 TL/OSL reader (Risoe DTU, Denmark). The reader is equipped with bialkali EMI 9235QB photomultiplier tube, which has maximum detection efficiency between 200 and $400 \mathrm{~nm}$. The Shott BG39 band pass filter enabling luminescence measurement in the range of 350-650 nm was used as the detection filter. TL glow curves were registered from room temperature up to $723 \mathrm{~K}\left(450^{\circ} \mathrm{C}\right)$ at heating rate of $5 \mathrm{~K} \cdot \mathrm{s}^{-1}$.

The samples under study were irradiated with various doses of alpha (Am-241, fluence $1.7 \cdot 10^{5} \mathrm{~cm}^{-2} \mathrm{~s}^{-1}$, energy at sample surface $4.5 \mathrm{MeV}$ ) and beta particles (Sr-90/Y-90, $\mathrm{E}_{\max }=2.3 \mathrm{MeV}$ ). The penetration depths of alpha particles in studied materials were calculated using SRIM software [14]. These depths for LSO, YSO, and LiF:Mg,Cu,P were $11.68 \mu \mathrm{m}$, $13.41 \mu \mathrm{m}$, and $16.73 \mu \mathrm{m}$, respectively. They are comparable with the thickness of SCFs (Table II). Beta particle dose is approximately uniform over the whole volume of the samples. Before each irradiation, samples were annealed for $240 \mathrm{~s}$ in $723 \mathrm{~K}\left(450^{\circ} \mathrm{C}\right)$ to erase any possible TL information. During all stages of experiments, the samples under study were kept in the darkness owing to their light sensitivity. 


\section{RESULTS AND DISCUSSION}

\section{A. Single Crystals Versus Single Crystalline Films}

As outlined in the introduction, a different crystalline form causes notable differences in TL properties of examined materials. Fig. 1 presents the comparison of TL response between SC and SCF LSO-based samples exposed to alpha particles. Although all samples were irradiated with the same fluence of alpha particles $\left(8.16 \cdot 10^{7} \mathrm{~cm}^{-2}\right)$, the mean deposited doses were not identical due to different penetration depth. For normalization, depths of alpha particles penetration layers were estimated and mean doses were calculated. The measured glow curves were normalized to unit dose $(1 \mathrm{~Gy})$ and to unit mass $(1 \mathrm{mg})$ of irradiated volume. As shown in Fig. 1(a), the LSO SC possesses significant TL in the range above room temperature with four clearly distinguishable peaks. The main peak position of this sample is at $381 \mathrm{~K}\left(108^{\circ} \mathrm{C}\right)$. The glow curve of LSO SCF does not possess the peak at $381 \mathrm{~K}$. Its main peak is localized at approximately $438 \mathrm{~K}\left(165^{\circ} \mathrm{C}\right)$ and has intensity over 10 times lower than its $\mathrm{SC}$ counterpart.

The LSO:Ce SC has main peak at $374 \mathrm{~K}\left(101^{\circ} \mathrm{C}\right)$, whereas it is not present in glow curve of its SCF analogue (Fig. 1b). The Ce-doped LSO SC and SCF have similar main peak positions as its undoped counterparts. The main difference between their TL glow- curves is the proportion between intensities of their peaks. It is important to note that the intensities of LSO:Ce SCF and SC main peaks are very high. Namely, LSO:Ce SC main peak is more than 4 times higher (1.4 times for LSO:Ce SCF) than that of the well-known MCP dosimetric material. However, it is important to mention that MCP efficiency for alpha radiation (which was used in this comparison) is about 20 times lower than for gamma or beta radiation.

The glow curves of undoped YSO SC and SCF (Fig. 2a) under the same alpha particles excitation, as in the case of LSO samples, show similar shape and main peak position $(435 \mathrm{~K}$, $162^{\circ} \mathrm{C}$ ), but slightly different intensities. The intensity of these peaks strongly increases under Ce doping, especially for SCF samples (Fig. 2b). The TL intensities of Ce-doped SCF and SC samples can be directly compared due to comparable content of $\mathrm{Ce}^{3+}$ ions in SC and SCF counterparts (see Tables I and II). The differences in intensities may be the result of both different growth method and slightly different ion concentration.

The undoped LSO and YSO SC and SCF samples show very different TL properties. The intensity of TL signal is significantly higher for LSO SC (five times) and YSO SC (also five times).

Above-mentioned results show that different methods of material preparation resulted in the different thermo-luminescent properties of undoped and $\mathrm{Ce}^{3+}$ doped YSO and LSO SC and SCFs. In case of undoped YSO and LSO SC and SCF samples, these differences can be caused by the presence or absence of main host defects (first of all, oxygen vacancies) as trapping centers. Namely, the low temperature method of LSO and YSO SCF preparation by LPE in oxygen containing atmosphere (air) usually results in the strong decreasing of the concentration of oxygen vacancies in them in comparison with $\mathrm{SC}$ counterparts [6], [15]. That correlates with the significantly lower intensity

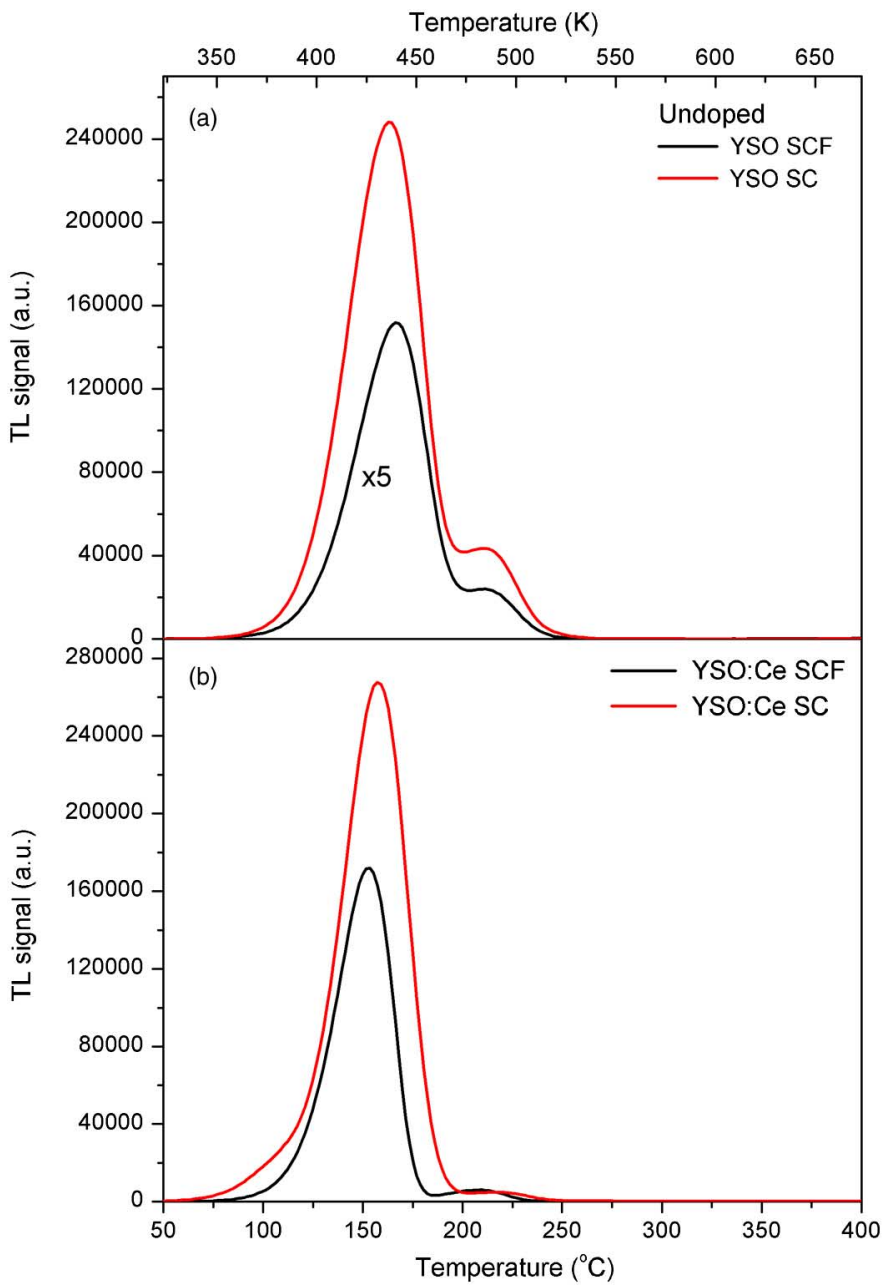

Fig. 2. Glow curves of undoped and Ce-doped YSO SC and SCF irradiated with alpha particles. All glow curves were normalized to unit mass of alpha penetration layer $(1 \mathrm{mg})$ and unit mean dose $(1 \mathrm{~Gy})$. TL signal of YSO SCF were multiplied by factor 5. (a) YSO SC (sample 25) and YSO SCF (sample 21). (b) YSO:Ce SC (sample 27) and YSO:Ce SCF (sample 24).

of TL of LSO and YSO SCFs with respect to SC analogues [Figs. 1(a) and 2(a), respectively].

Another set of trapping centers can be formed in the $\mathrm{Ce}^{3+}$ doped YSO and LSO based SCFs. Due to use of the PbO-based flux for SCF preparation by LPE method, the $\mathrm{Pb}^{2+}$ ions are incorporated in these SCFs in concentration even up to tenth at.\% (see [8]-[10] for details). Recently we found the strong negative influence of the $\mathrm{Pb}^{2+}$ flux related dopant on the scintillation light yield (LY) of LSO:Ce and YSO:Ce SCFs [8], [10]. Namely, the YSO:Ce and LSO:Ce SCFs samples, grown from the PbO-based flux, show the LY about $25-40 \%$ of that for reference LYSO:Ce SC under excitation by $\alpha$-particles of Pu-239 source. The absorption spectra of these SCF samples show the strong wide band peaked approximately at $250-260 \mathrm{~nm}$ range which most probably related to creation of the $\mathrm{Pb}^{2+}-\mathrm{Ce}^{4+}$ pair centers [16]. The presence of such centers was found also in $\mathrm{Ca}^{2+}$ co-doped LuYSO:Ce SC [16]. Thus, the Ce doped YSO and LSO SCFs can possess a large concentration of trapping centers related to the mentioned $\mathrm{Pb}^{2+}-\mathrm{Ce}^{4+}$ pairs. This assumption explains the 1) strong increase of the TL intensity in YSO:Ce and LSO:Ce SCFs with respect to their undoped SCF 


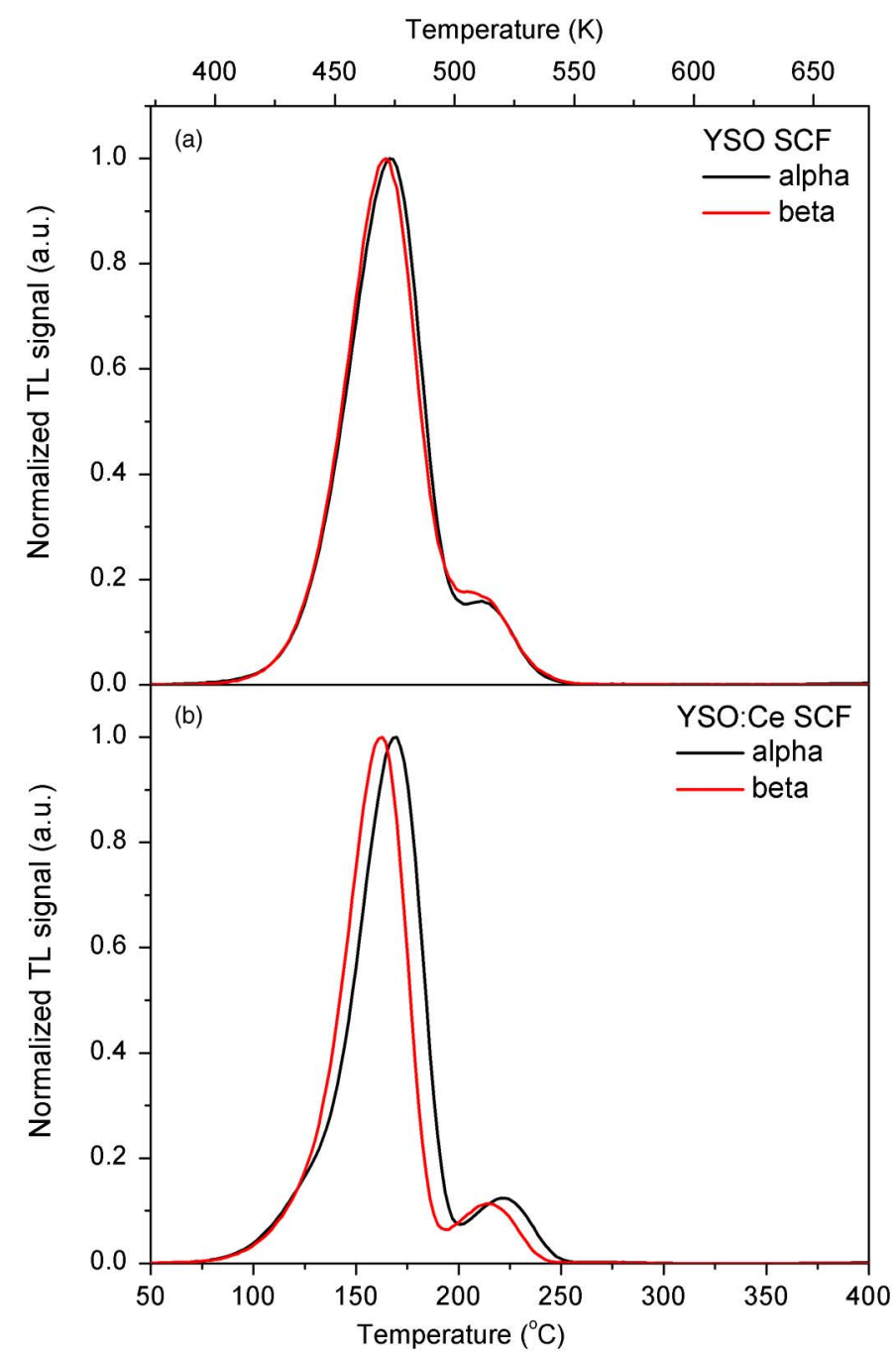

Fig. 3. Comparison of glow curves of undoped and Ce-doped LSO samples after alpha and beta irradiation. All glow curves were normalized to 1. (a) LSO SCF (sample 1). (b) LSO:Ce SCF (sample 3).

counterparts and 2) the completely different shape of the glow curves for SC and SCF of these silicates (Figs. 1 and 2).

It is important to note here that nominally undoped $\mathrm{YSO}$ and LSO SC samples can also contain the $\mathrm{Ce}^{3+}$ ions as trace impurity, which can play the role of recombination centers for electrons liberated from trapping centers, namely, from oxygen vacancies.

\section{B. Alpha Versus Beta Irradiation}

Fig. 3 presents the comparison of TL glow curves of LSO SCFs after alpha and beta irradiation. Annealed samples were irradiated with alpha and beta particles. The measured TL glow curves were normalized to the range $[0 ; 1]$. As already mentioned, the penetration depth of alpha particles in YSO and LSO is comparable with SCF thickness. With good approximation, it can be therefore assumed that signal after alpha irradiation originates only from SCF layer and not from a substrate, whereas response after beta irradiation comes from the whole sample (SCF + substrate). This way we can compare TL glow curves from SCF and substrate.

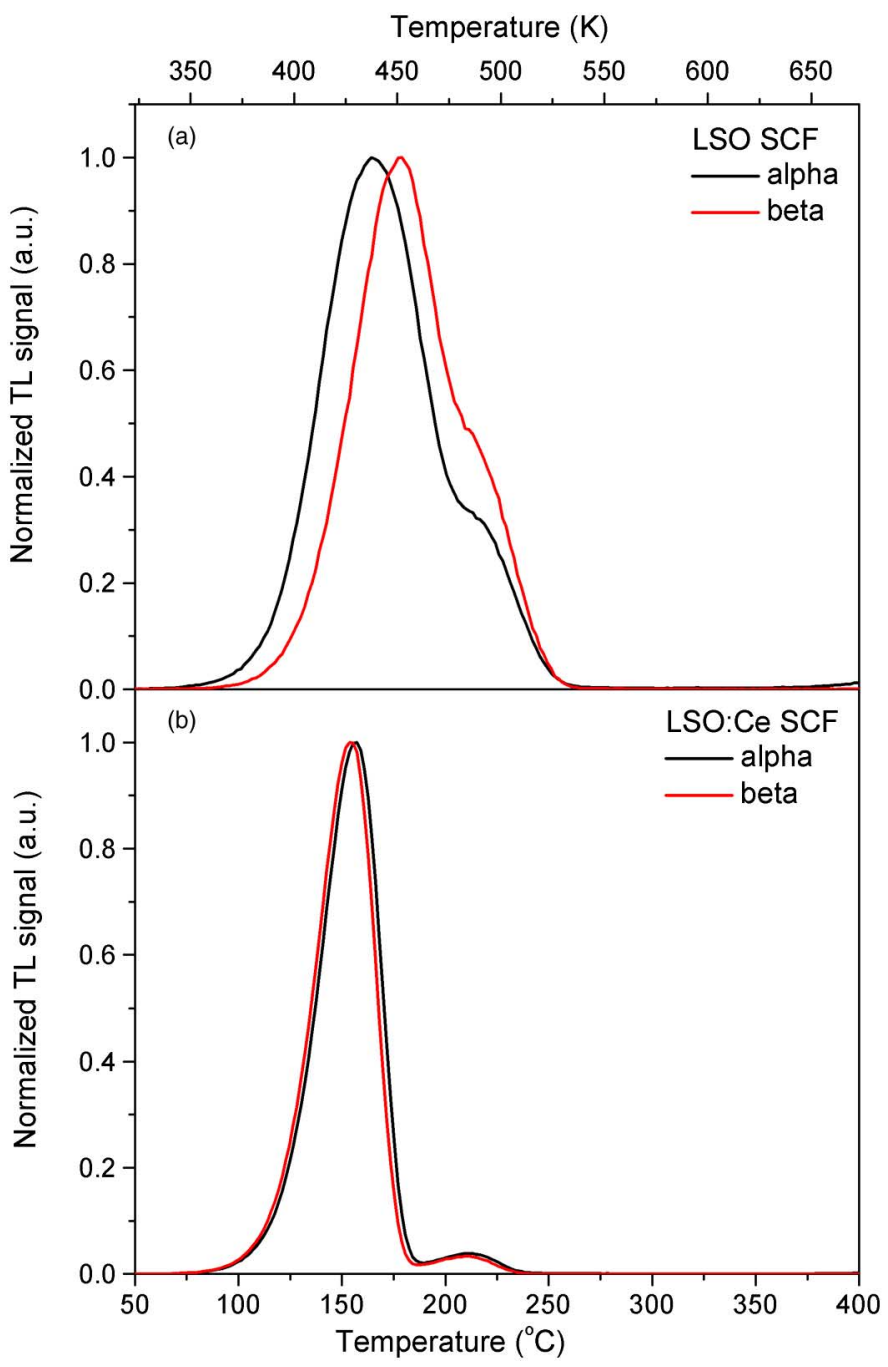

Fig. 4. Comparison of glow curves of undoped and Ce-doped YSO samples after alpha and beta irradiation. All glow curves were normalized to range $[0 ; 1]$. (a) YSO SCF (sample 21). (b) YSO:Ce SCF (sample 57).

As can be seen in Fig. 3(a), the main difference in TL glow curves of LSO SCF after beta and alpha irradiation is peak intensities ratio. Ce-doped LSO SCF shows no clear differences between the corresponding glow curves [Fig. 3(b)].

Comparison of undoped and Ce-doped YSO SCFs shows some differences in TL glow curves excited with alpha and beta particles (Fig. 4). In particular, the proportion between two distinguishable peaks is different after alpha and beta irradiation, although the trend is opposite when considering undoped and doped samples. For undoped YSO samples, the intensity of the second peak is higher after irradiation with beta particles [Fig. 4(a)], and vice versa for Ce-doped YSO [Fig. 4(b)].

\section{Single Crystalline Film Versus Substrate}

To establish which part of TL signal originates from SCF and which from the substrate, the comparison of TL behavior of two SCF samples and their substrates was made. Samples under study were irradiated with the same fluence of alpha particles and read out in the same conditions. For comparison, the achieved results were normalized using the procedure presented in Section A. 


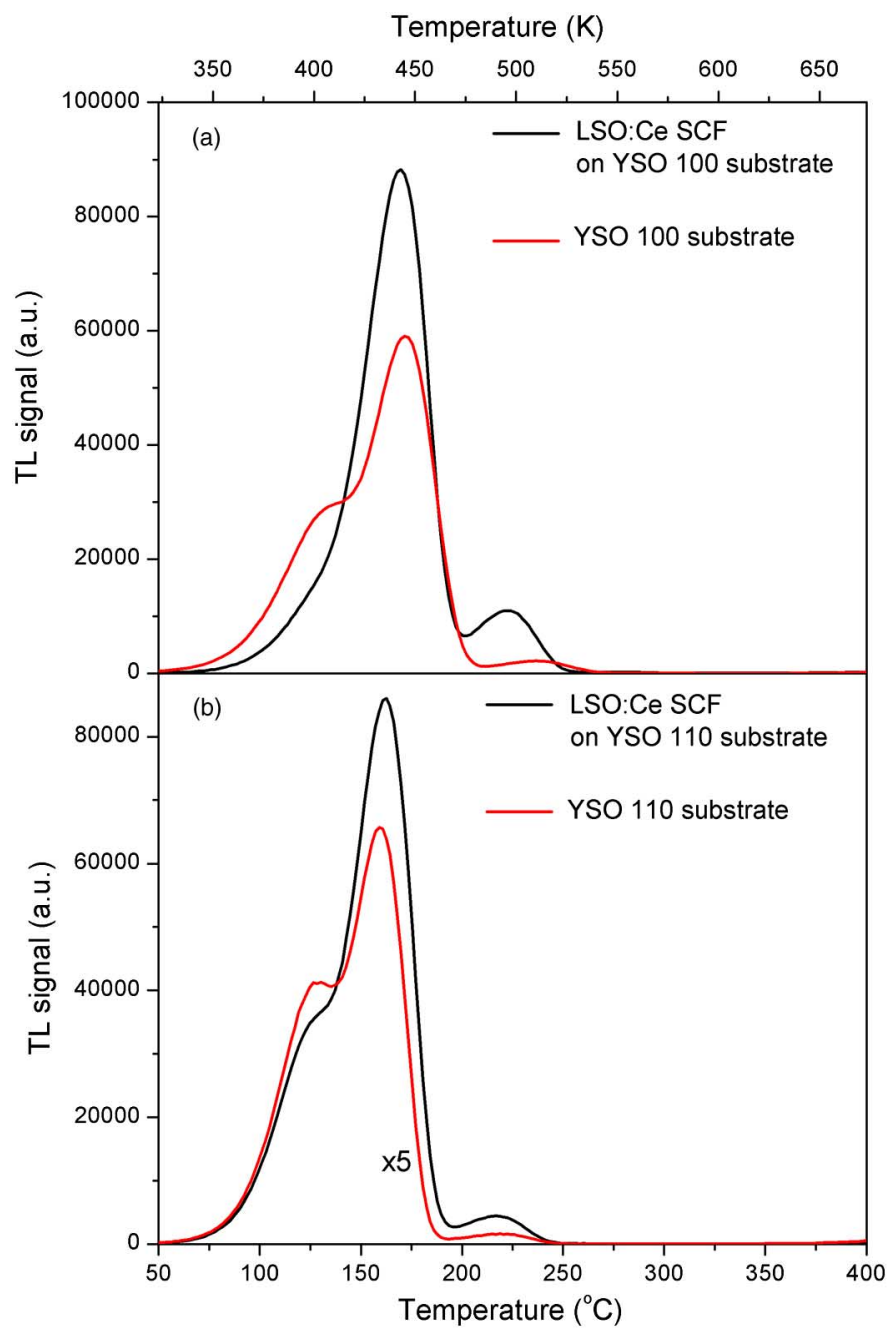

Fig. 5. TL glow curves of LSO:Ce SCF samples compared with their substrates. The results ware normalized unit mass $(1 \mathrm{mg})$ and unit mean dose (1 Gy). TL signal of YSO 110 substrate were multiplied by factor 5. (a) LSO:Ce SCF based on YSO 100 substrate (sample 9) and YSO 100 substrate (sample 11). (b) LSO:Ce SCF based on YSO 110 substrate (sample 10) and YSO 110 substrate (sample 12).

It can be clearly seen from Fig. 5 that TL behavior of SCFs and their substrates are different. The TL intensity of LSO:Ce SCF sample based on YSO 100 substrate is significantly higher than that of its substrate (over 1.5 times). The difference is even stronger in case of the high-temperature peak, which is almost absent for the substrate [Fig. 5(a)]. In case of the second set of the samples (LSO:Ce SCF based on 110 substrate and its substrate), differences in intensities are significantly bigger (intensity of the substrate is by more than 6 times lower). In addition, as Fig. 5(b) shows, the disproportion of the high temperature peak is stronger. In both cases $(a, b)$, low temperature peak occurred in TL glow curves. It can be observed that this peak originates from substrates.

Most probably, the difference in the TSL intensity of LSO:Ce SCF with (100) and (110) orientation (Fig. 5) are caused by the different concentration of the $\mathrm{Ce}^{3+}$ emission centers in them.

\section{LSO and YSO-Based Orthosilicates Versus MCP}

The intensities of luminescence of the studied samples were compared with the luminescent output of the $\mathrm{LiF}: \mathrm{Mg}, \mathrm{Cu}, \mathrm{P}$
TABLE III

TL RESPONSE OF LSO AND YSO SAMPLeS AFTER ALPHA AND BETA IRRADIATION COMPARED TO THE STANDARD LithIUM FLUORIDE MCP TL DETECTORS

\begin{tabular}{|c|c|c|c|c|}
\hline \multirow{2}{*}{$\begin{array}{l}\text { Sample } \\
\text { number }\end{array}$} & \multicolumn{2}{|c|}{ Beta response } & \multicolumn{2}{|c|}{ Alpha response } \\
\hline & Integral & Peak height & Integral & Peak Height \\
\hline 1 & 0.742 & 0.683 & 0.33 & 0.36 \\
\hline 2 & 1.135 & 1.360 & 0.81 & 1.30 \\
\hline 3 & 0.740 & 1.194 & 0.58 & 1.20 \\
\hline 4 & 0.689 & 0.732 & 0.42 & 0.59 \\
\hline 5 & 0.660 & 1.054 & 0.74 & 1.50 \\
\hline 6 & 1.089 & 1.349 & 0.87 & 1.40 \\
\hline 7 & 0.789 & 1.271 & 0.55 & 1.13 \\
\hline 8 & 0.498 & 0.805 & 0.48 & 1.00 \\
\hline 9 & 0.449 & 0.611 & 0.63 & 1.05 \\
\hline 10 & 0.270 & 0.370 & 0.65 & 1.03 \\
\hline 16 & 0.904 & 0.635 & 4.07 & 4.08 \\
\hline 17 & 0.001 & 0.000 & 0.02 & 0.01 \\
\hline 18 & 0.004 & 0.002 & 0.05 & 0.04 \\
\hline 19 & 0.275 & 0.329 & 1.61 & 2.70 \\
\hline 21 & 0.852 & 1.027 & 0.23 & 0.36 \\
\hline 22 & 0.849 & 1.393 & 0.37 & 0.70 \\
\hline 23 & 0.832 & 0.759 & 0.13 & 0.14 \\
\hline 24 & 0.965 & 1.584 & 0.98 & 2.05 \\
\hline 56 & 0.508 & 0.689 & 0.53 & 0.89 \\
\hline 57 & 0.705 & 0.983 & 0.78 & 1.33 \\
\hline 25 & 1.169 & 1.424 & 1.96 & 2.97 \\
\hline 11 & 0.181 & 0.237 & 0.52 & 0.70 \\
\hline 12 & 0.028 & 0.034 & 0.11 & 0.16 \\
\hline 26 & 0.705 & 0.683 & 1.60 & 1.81 \\
\hline 27 & 0.462 & 0.736 & 1.76 & 3.19 \\
\hline МСР & 1 & 1 & 1 & 1 \\
\hline
\end{tabular}

(MCP), which is, as previously mentioned, a standard TL dosimetric material. The samples were irradiated with both alpha and beta particles. To compensate differences of masses shapes and sizes of investigated samples, the results had to be normalized. In case of beta irradiation, TL signal was calculated for $1 \mathrm{mg}$ of whole sample. The results of TL readout after excitation with alpha particles were as presented before. The results were then normalized to MCP response. Results are presented in Table III. The integral value was calculated as the sum of TL signal in whole readout range. The peak height value is the intensity of the highest peak in investigated glow curve.

As can be seen from Table III, the TL intensity of most of LSO and YSO orthosilicates is comparable or even higher than that of MCP. After beta irradiation, LSO:Ce SCF samples show the highest intensity, both for integral and main peak height. Larger differences between response of silicates and MCP can be seen after exposure to alpha particles, but it should be noticed that MCP alpha efficiency is only about $5 \%$ in comparison to its efficiency for beta particles [12].

\section{CONCLUSIONS}

We perform in this work the comparative analysis of thermoluminescent properties of undoped and Ce-doped $\mathrm{Y}_{2} \mathrm{SiO}_{5}$ and $\mathrm{Lu}_{2} \mathrm{SiO}_{5}$ single crystals and single crystalline films under $\alpha$ - and $\beta$-particles excitation. The SCs were grown by the Czochralski method, and SCFs were grown by the liquid phase epitaxy method from PbO-based melt-solutions.

We show that differences in the methods of material preparation resulted in the different thermoluminescent properties 
of undoped YSO and LSO SC and SCF. Such differences are caused by the presence or absence of main host defects (first of all, oxygen vacancies) as trapping centers. Namely, the undoped YSO and LSO SCs, possess large concentration of oxygen vacancies, which can play the role of deep trapping centers. On the contrary, the concentration of oxygen vacancies is strongly reduced in SCF due to low temperature of SCF crystallization by LPE method.

In $\mathrm{Ce}^{3+}$ doped YSO and LSO SCF we presume the creation of the $\mathrm{Ce}^{4+}-\mathrm{Pb}^{2+}$ pair centers. Such type of centers can be responsible for the strong increasing of TL intensity of LSO:Ce and YSO:Ce SCF in comparison with that for SC counterparts. The intensity of TL of LSO:Ce and YSO:Ce SCFs is comparable with conventional dosimetric material $\mathrm{LiF}: \mathrm{Mg}, \mathrm{Cu}, \mathrm{P}(\mathrm{MCP})$.

\section{REFERENCES}

[1] M. Globus and B. Grinyov, Non-Organic Scintillators. New and Traditional Materials. Kharkiv, Ukraine: Akta, 2000.

[2] C. L. Melcher and J. S. Schweizer, "Ce doped lutetium orthosilicates: A fast, efficient new scintillators," IEEE Trans. Nucl. Sci., vol. 39, pp. 502-505, 1992.

[3] C. L. Melcher, R. A. Manente, C. A. Peterson, and J. S. Schweizer, "Czochralski growth of rare earth oxyorthosilicate single crystals," $J$. Cryst. Growth, vol. 128, pp. 1001-1005, 1993.

[4] G. Ren, L. Qin, L. S. Lu, and H. Li, "Scintillation characteristics of lutetium oxyorthosilicate," Nucl. Instrum. Meth. Phys. Res. A., vol. 531, pp. 560-565, 2004.

[5] T. Martin and A. Koch, "Recent development in X-ray imaging with micrometer spatial resolution," J. Synchrotron Rad., vol. 13, pp. 180-194, 2006

[6] T. Martin, P.-A. Douissard, M. Couchaud, A. Cecilia, T. Baumbach, K. Dupré, and A. Rack, "LSO-based single crystal film scintillator for synchrotron-based hard X-ray micro-imaging," IEEE Trans. Nucl. Sci., vol. 56, pp. 1412-1418, 2009.

[7] P.-A. Douissard, A. Cecilia, T. Martin, V. Chevalier, M. Couchaud, T. Baumbach, K. Dupré, M. Kühbacher, and A. Rack, "A novel epitaxially grown LSO-based thin-film scintillator for micro-imaging using hard synchrotron radiation," J. Synchrotron Rad., vol. 17, pp. 571-583, 2010.
[8] Y. Zorenko, M. Nikl, V. Gorbenko, V. Savchyn, T. Voznyak, R. Kucerkova, O. Sidletskiy, B. Grynyov, and A. Fedorov, "Growth and luminescent properties of $\mathrm{Lu}_{2} \mathrm{SiO}_{5}$ and $\mathrm{Lu}_{2} \mathrm{SiO}_{5}: \mathrm{Ce}$ single crystalline films," Opt. Mater., vol. 33, pp. 846-852, 2011.

[9] Y. Zorenko, V. Gorbenko, V. Savchyn, T. Voznyak, B. Grinyov, O. Sidletskiy, D. Kurtsev, A. Fedorov, V. Baumer, M. Nikl, J. A. Mares, A. Beitlerova, and M. Kucera, "Growth and luminescent properties of $\mathrm{Lu}_{2} \mathrm{SiO}_{5}$ : $\mathrm{Ce}$ and $\left(\mathrm{L}_{1-\mathrm{x}} \mathrm{Gd}_{\mathrm{x}}\right)_{2} \mathrm{SiO}_{5}: \mathrm{Ce}$ single crystalline films," J. Crystal Growth, vol. 337, pp. 72-80, 2011.

[10] Y. Zorenko, V. Gorbenko, V. Savchyn, T. Voznyak, V. V. Gorbeno, M. Nikl, J. A. Mares, O. Sidletskiy, B. Grynyov, A. Fedorov, K. Fabisiak, and K. Paprocki, "Scintillation and luminescent properties of undoped and $\mathrm{Ce}^{3+}$ doped $\mathrm{Y}_{2} \mathrm{SiO}_{5}$ and $\mathrm{Lu}_{2} \mathrm{SiO}_{5}$ single crystalline films grown by LPE method," Opt. Mater., vol. 34, pp. 1969-1974, 2012.

[11] A. Twardak, P. Bilski, Y. Zorenko, V. Gorbenko, A. Mandowski, E. Mandowska, and O. Sidletskiy, "Comparative study of TSL and OSL properties of LSO and LSO:Ce single crystals and single crystalline films," Radiat. Meas., vol. 56, pp. 196-199, 2013.

[12] P. Bilski, P. Olko, B. Burgkhardt, E. Piesch, and M. P. R. Waligorski, "Thermoluminescence efficiency of $\mathrm{LiF}: \mathrm{Mg}, \mathrm{Cu}, \mathrm{P}(\mathrm{MCP}-\mathrm{N})$ detectors to photons, beta-electrons, alpha-particles and thermal-neutrons," Radiat. Prot. Dosim., vol. 55, pp. 31-38, 1994.

[13] E. Mandowska, P. Bilski, B. Obryk, A. Mandowski, P. Olko, and J. Kim, "Spectrally resolved thermoluminescence of highly irradiated LiF:Mg, $\mathrm{Cu}, \mathrm{P}$ detectors," Radiat. Meas., vol. 45, pp. 579-582, 2010

[14] J. F. Ziegler, M. D. Ziegler, and J. P. Biersack, "SRIM - the stopping and range of ions in matter," Nucl. Instrum. Meth. Phys. Res. B, vol. 268, pp. 1818-1823, 2010.

[15] Y. Zorenko, T. Zorenko, V. V. Gorbenko, T. Voznyak, V. Savchyn, P. Bilski, and A. Twardak, "Peculiarities of luminescent and scintillation properties of YAG:Ce phosphor prepared in different crystalline forms," Opt. Mater., vol. 34, pp. 1969-1974, 2012.

[16] W. Chewpraditkul, C. Warnak, T. Szczesniak, M. Moszcynski, V. Jari, A. Beitlerova, and M. Nikl, "Comparison of the absorption, luminescence and scintillation characteristic in $\mathrm{Lu}_{1.95} \mathrm{Y}_{0.05} \mathrm{SiO}_{5}$ : Ce, Ca and $\mathrm{Y}_{2} \mathrm{SiO}_{5}$ scintillators," Opt. Mater., accepted for publication.

[17] R. Visser, C. L. Melcher, J. S. Schweizer, H. Suzuki, and T. A. Tombrello, "Photostimulated luminescence and thermoluminescence of LSO scintillators," IEEE Trans. Nucl. Sci, vol. 41, pp. 689-693, 1994. 